\title{
SARS-CoV-2 pandemic in Italy: ethical and organizational considerations
}

\author{
Stefano Nardini, ${ }^{1}$ Claudio M. Sanguinetti, ${ }^{1}$ Fernando De Benedetto, ${ }^{1}$ Claudio Baccarani, ${ }^{2}$ Mario Del Donno,, 3 \\ Mario Polverino, ${ }^{1,4}$ Isabella Annesi-Maesano ${ }^{5}$ \\ ${ }^{1}$ Italian Respiratory Society Research Centre, Milan, Italy \\ ${ }^{2}$ Business Management, University of Verona, Italy \\ ${ }^{3}$ Pneumology Unit, A.O.R.N. "San Pio"-P.O. “G. Rummo”, Benevento, Italy \\ ${ }^{4}$ Endoscopic Unit, Pulmonary Division, Hospital M. Scarlato, Scafati (SA), Italy \\ ${ }^{5}$ INSERM and Epidemiology of Allergic and Respiratory Diseases Department, Sorbonne University, IPLESP, Paris, France
}

The current SARS-CoV-2 pandemic is still raging in Italy. The country is currently plagued by a huge burden of virusrelated cases and deaths. So far, the disease has highlighted a number of problems, some in common with other Countries and others peculiar to Italy which has suffered from a mortality rate higher than that observed in China and in most Countries in the world. The causes must be sought not only in the average age of the population (one of the oldest in the world), but also in the inconsistencies of the regional health systems (into which the National Health System is divided) and their delayed response, at least in some areas. Ethical issues emerged from the beginning, ranging from restrictions on freedom of movements and restrictions on personal privacy due to the lockdown, further to the dilemma for healthcare professionals to select people for ICU hospitalization in a shortage of beds in Intensive Care Unit (ICU). Organizational problems also emerged, although an official 2007 document from the Ministry of Health had planned not only what measures had to be taken during an epidemic caused by respiratory viruses, but also what had to be done in the inter-epidemic period (including the establishment of DPIs stocks and ventilators), vast areas of Italy were totally unprepared to cope with the disease, as a line of that document was not implemented. Since organizational problems can worsen (and even cause) ethical dilemmas, every effort should be made in the near future to prepare the health system to respond to a similar emergency in a joint, coherent, and homogeneous way across the Country, as planned in the 2007 document.

In this perspective, Pulmonary Units and specialists can play a fundamental role in coping with the disease not only in hospitals, as intermediate care units, but also at a territorial level in an integrated network with GPs.

Key words: SARS-CoV-2; mortality rate; ICU beds; ethical and organizational issues; intermediate respiratory care.

Correspondence: Stefano Nardini, Italian Respiratory Society Research Centre, Via San Gregorio 12, 20124 Milano, Italy. E-mail: snardini.pneumologo@gmail.com

Contributions: All the authors made a substantive intellectual contribution, read and approved the final version of the manuscript and agreed to be accountable for all aspects of the work.

Conflict of interest: The Authors declare no conflict of interest for this paper. CMS is Editor-in-Chief of Multidisciplinary Respiratory Medicine.

Funding: This study has not received any specific grant by anyone. 


\section{Introduction}

There is no doubt that the SARS-CoV-2 epidemic has changed a lot in our lives and will change even more in the future. Across the world, the epidemic has shown some degree of unpreparedness for infectious emergencies and-during the first phase of the emergency - a dangerous unresolved reaction, due to financial problems and budgetary considerations [1].

The spread of the virus has not been (and actually is not) the same throughout Italy and probably many causes are to be considered, organizational [1] as well as genetical ones but the following facts are surely shared nationwide. Some limits are inherent in the organization of the National Health Service (NHS). First, the gigantic mistake of dividing the National Health Service into over 21 different regional services, a division that has hindered consistency in the fight against the epidemic. For instance, the political decision to initially block only some regions of Northern Italy caused a massive exodus to Southern Italy, thus contributing to the spread of the epidemic, even to a small extent in non-infected regions. Secondly, the progressive underbudgeting of the NHS, with a reduction in the availability of hospital beds without a real and substantial increase in extra-hospital facilities. Thirdly, the limitations of an NHS model shaped near only on the prevention and treatment of chronic non-communicable chronic conditions, neglecting the practical organization of emergencies (not uncommon during the normal winter flu season). Fourth, the minor role and the ranking given in last years to the Pulmonary Units and Clinics in the organization of the Italian NHS: the respiratory units have been conceived as spare Internal Medicine Unit and the specialists as maimed internist, useless out of endoscopy. This downsizing has been attributed to both the weakness of Universities and Respiratory Scientific Societies. A role completely disproportionate to the prevalence and burden of pulmonary disease and to the misunderstanding of the potential place of Respiratory Medicine inside and outside hospitals. On the whole, this Covid-19 crisis has highlighted a fragility of both the economic and financial system as well as the Health system.

The present pandemic has already given importance to smart working and digital transformation and will certainly accelerate the introduction of tele-medicine (i.e., devices and skills that allow real-time interactive two-way communication between patients and healthcare personnel, even if distant sites) especially in respiratory medicine. However, tele-medicine in Italy is not included in the "essential levels of assistance" (i.e., the definition of a set of services defined at National level, to be guaranteed at local level). If this pandemic will at least lead the Italians to deal with the problems mentioned above, will be a positive result, unfortunately obtained at a very high price.

This paper, after considering the current situation (compared to previous health emergencies) and the consequent clinical and ethical problems, describes the changes that seem advisable in the near future to prepare the NHS not only for the immediately following phase of the pandemic, but also in the long term to face another predictable pandemic originating from a respiratory virus. In fact, we can imagine that the challenge in the near future will be to face the consequences of globalization: not only well-known climatic emergencies, but also emergencies of new pandemics.

In 2007, when the spread of avian influenza A (H5N1) was expected, the Italian National Center for Disease Control (CCM) produced a document to cope with flu pandemics [2]. Actions for each phase of a pandemic have been recommended in this document, including recommendations for "interpandemic" periods and, among other things, all measures have been identified to be ready for another pandemic (including adequate DPIs stock, of mechanical ventilators and ICU beds).
Regarding the respiratory field, in a paper published and produced at the end of 2009 on behalf of the Interdisciplinary Scientific Association for the Study of Respiratory Diseases (AIMAR) [3], a protocol was developed for the management of pandemic influenza A (H1N1) - 2009 in respiratory units. This document defined the role and competences specific for the respiratory units and specialists, aiming at designing their functions within the actions envisaged for the entire Italian NHS [2].

The influenza A (H1N1) pandemic began in March 2009 in Mexico, was declared a pandemic by the WHO in June, peaked between October and November and was exhausted by August of the following year [4]. It should be noted that the period was somewhat different from the current one and, in fact, it was a change of a known virus, against which there was a vaccine and the whole population, or at least the elderly, already had a certain immunity. The severe complications were mainly in youth (many serious and fatal cases were between 30 and 50 years old) and in pregnant women and symptoms were involved not only in the respiratory tract, but also in the gastrointestinal tract. Furthermore, there have been effective treatments with neuraminidase inhibitors, at least when given in the first 48 hours of symptoms. On the other hand, it must be considered that SARS-CoV-2 seems much more infectious than A (H1N1) - 2009, since it spread worldwide in less than three months, probably due to the increase of mobility of people across the world, which has increased significantly in the past decade. Finally, while vaccines for A (H1N1) - 2009 had already been developed in September 2009, a vaccine for SARS-CoV-2 is not ready yet. However, there were also a few similarities as demonstrated in a high percentage level of severe lung lesions of SARS-CoV-2, present in A (H1N1) too. People suffering from cardiac and chronic respiratory diseases or diabetes were at greater risk of severe complications and death. Care facilities (including GPs, A\&E units, medical and Pulmonary wards) somewhere were almost overwhelmed by the number of patient admissions.

The aim of this paper is to highlight some challenges that are being faced during the current pandemic and, comparing it with that of 2009, obtain a part of useful advice for better management of this and possible new pandemics.

\section{The present pandemic}

Between the last months of 2019 and the beginning of 2020 , China at first and currently Italy too, made a strong commitment to face a very difficult situation, a problem consisting of a widespread infection induced by a new coronavirus named SARS-CoV-2. The International Committee recommended this denomination for the Taxonomy of Viruses because of the similarity, but not the identity, of the new virus with the viral agent that caused the original acute severe respiratory syndrome (SARS) at the beginning of this century, in the Chinese region of Guandong and spread rapidly all over the world.

The surprising feature of this new coronavirus consists in its marked ability to diffuse among individuals with greater rapidity and capacity of infection than that of seasonal flu and other respiratory viral infections, with the exception of that of measles, which has one of the highest. Therefore, the basic reproductive rate of SARS-CoV-2 (how many people can be infected by a positive individual) was estimated at the beginning between 2 and 3 and now just around 1. This is probably the main reason (together with an underestimate during the first phase) for which the virus spread so widely in China and then in many other countries of the world, giving rise to a COVID-19 outbreak recognized as a pandemic by the WHO [5]. At the time of writing this paper (April 6, 2020), 120,290 infected people are registered in Italy, with 14,381 deaths 
[6], consulted as of April 6. That is a crude fatality rate of $12.32 \%$, higher than that observed in China, where 82,930 infected cases were recorded, with 3,338 deaths [7]. So in Italy, the infection spread very fast and caused more deaths.

In general, the reasons for the spread of disease is the lack of a vaccine and herd immunity, while the lack of specific treatments partially explains the unfavorable effects of the disease. Some antiviral drugs already tested and proven effective against other viral infections such as SARS, MERS (Middle Eastern Respiratory Syndrome of 2012) and HIV are also used in COVID-19, with encouraging results in some cases where they have been administered as compassionate use, before adequate and well-designed studies are performed. Other treatments are also being tested in SARS-CoV-2 pneumonia, including hydroxychloroquine, passive antibody transfer from convalescent patients, drugs that block the ACE2 receptor to reduce inflammation, monoclonal antibodies and even mesenchymal stem cells to counteract excessive inflammation [8]. For the specific Italian situation, the rapid spread could be explained by undocumented infections before the implementation of travel and movement restrictions: in China these undocumented infections caused mild symptoms and went mainly underestimated [9].

Another factor that appears to have played a role that could contribute to the spread of the virus, at least in the beginning, was the fact that a large number of people, who suspected of having symptoms related to SARS-CoV-2 disease, went to the emergency room or own doctors, without any protection. It is to be noted that, even in ordinary, non-epidemic times in Italy it is customary for patients, disregarding the mildness or severity of their symptoms, to access the A\&E department (with the so-called "white codes"). Obviously, many patients were also visited during the first phases of the pandemic period when no preventive barriers were operating, to the detriment of many other patients and healthcare facilities. Finally, it can be considered the fact that asymptomatic patients (obviously not detected) can convey the disease [10]. Even now, if separate lanes for SARS- and non-SARS patients are not present, hospitals can act as hubs for the spread of epidemics.

In fact, according to Chinese figures [11], COVID-19 has a favorable course in the vast majority of cases, while in $5 \%$ of patients intensive care is needed, with invasive ventilation and high probability of fatal outcome, especially when patients are already affected with other chronic diseases. For the specific Italian high rate of deaths, a certain number of explanations have been proposed. In our opinion under the high number of cases (spread) and deaths (unfavorable outcomes), there were three factors, differently operating in different areas of our Country. The first one has probably been the fact that in Italy there is a great percentage of older age population (individuals $\geq 65$ years old), some of them suffering from chronic conditions and, consequently, more exposed to serious complication and death from this coronavirus [12]. The aged population (i.e., over 65), accounts in our country for nearly one fourth of the whole population and is expected to further increase so as to reach the $34 \%$ by the next 25 years [13]. The second factor could be that the lockdown in some areas was decided very late, due to economic considerations. Finally, when the epidemic was officially recognized, sick people have been requested to stay at home, waiting for an evaluation. May be that patients remained at home in the following days, even if becoming severely ill, eventually came to health facilities only when the disease was at very severe stages, often requiring ICU (actually, if demonstrated, this outcome further emphasizes the importance of tele-medicine). Taking into consideration that this disease can evolve to respiratory failure in a very short time.

The situation is not be changing in the short term, although treatments are being attempted, none of them so far, with evidence of effectiveness. Similarly, the availability of a specific vaccine against SARS-CoV-2 should not be reasonably expected before at least a few months, although several laboratories, in Italy too [4], have already started working to carry out this task in the minor possible time. To counter this bad situation, in such rapid evolution, China first and Italy subsequently, have adopted drastic decisions in order to decrease the continuously increasing curve of infections. Since no pharmacological treatments or approved vaccines are currently available, only one set of non-pharmacological interventions (NPI) [14] can be implemented. In particular, besides important hygienic measures like accurate hands washing and sanitization of things and environment, isolation of the case (the infected person remains at home, monitored), home quarantine (a contact person stays at home for 14 days), social distance of the entire population (when people away from home interact at a distance of not less than 1 meter), closure of schools, universities and workplaces (the latter except for the essential activity), and strict

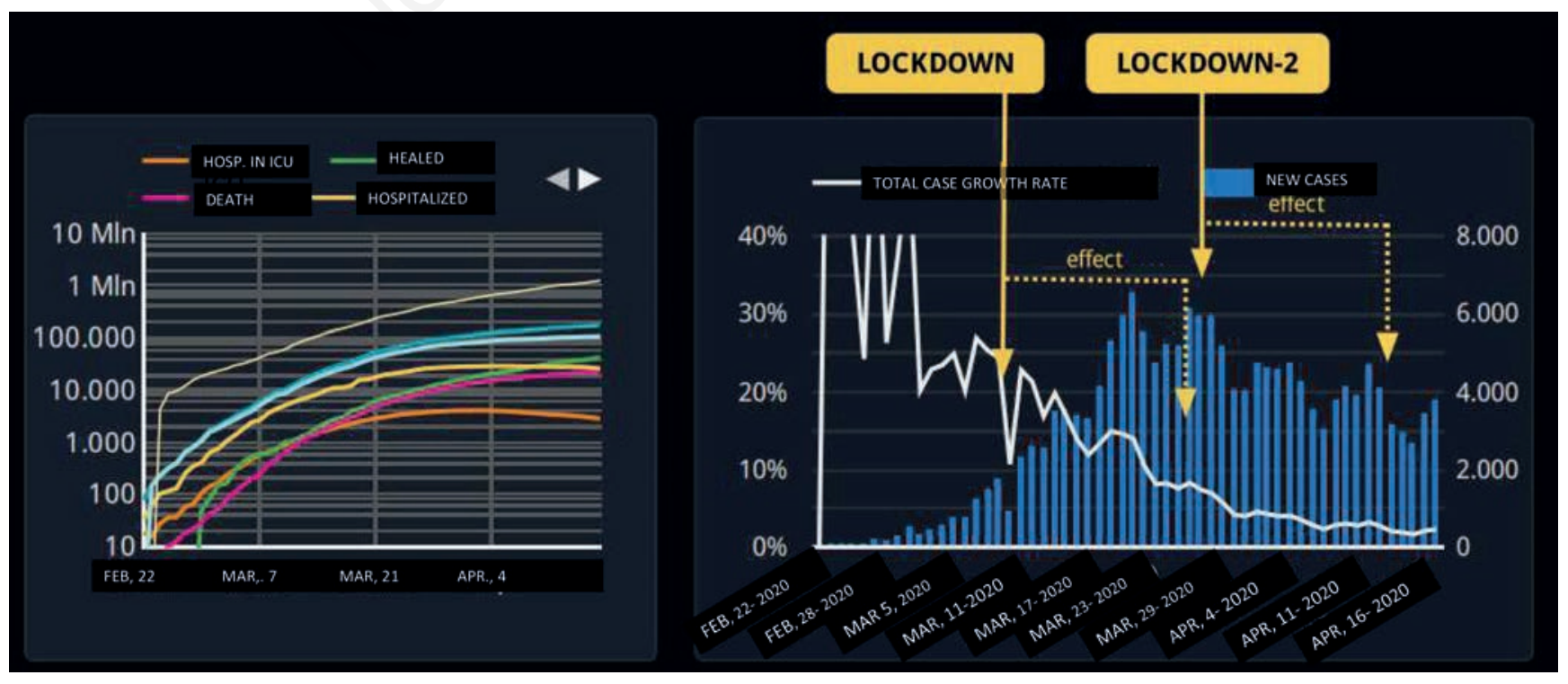

Figure 1. Pandemic trends in Italy from February 22 to April 162020 and lockdown effects. From https://covidtrends.com/ 
limitations of sport activities, in particular team activities. Indeed, these NPIs have been implemented in both China and Italy (Figure 1) [15], and even the complete blockade of about 60 million people has been adopted in a Chinese province. We do not know how these preventive measures were received in China, in Italy many citizens have found it difficult to understand some regional and national political leaders, fluctuating between the denial of the severity of the threat and the fear of its deadly consequences but always seeking media visibility.

\section{Ethical considerations}

To deal with the current epidemic in Italy, the entire population was initially brought to enforce social isolation; therefore, a more severe form of social distancing was implemented in a national block: the infected people were isolated and their contacts quarantined. Quarantine lasts for a time identified to correspond to the incubation period of the infection during which it could turn into disease. In Italy, as previously in China, entire regions or cities, besides all known infected individuals, have been subjected to the restrictive measures described above in an attempt to minimize contagion.

Lockdown and social distancing and similar public health surveillance, have been known for decades [16], but they can raise some ethical problems. Among the numerous public health surveillance activities, three main tasks should be highlighted: systematic collection of data relating to a specific disease, monitoring and evaluation of the data obtained and forwards reports to the institutions responsible for drawing up and implementing the action plans [17]. And, last but not least, the strict validation and comparison among the different methods presently used in collecting swabs and processing biological specimen. Particularly, in the field of infectious disease, ethics deals with the adequate personal behavior of individuals, correct work organization and public health decisions, aimed at reaching and maintaining the maximum degree of the control of an infectious disease [18].

Obviously, when important and restrictive decisions like quarantine or personal movement tracking are undertaken by the responsible for the public health surveillance, it is not unlikely that conflicts between the individual interests and the good of the community arise [19]. In this case, the question may arise whether it is more important to respect human rights and privacy or to use any way to promote public health. The answer to this question depends on the actual importance of the threat to the health of the community and on the availability of other measures that can counteract the spread of the infection.

This is what is now happening in Italy, given the current lack of an effective therapy or vaccine, thus the social isolation, while unpleasant, is the only effective mean and it should be respected by all citizens in the right perspective of individual and also collective benefit. Unfortunately, the entire population does not always comply with rules about isolation. Sometimes people give preference to their usual behavior and interest, without understanding or neglecting the real severity of the situation (up to $6 \%$ of controlled people fined in a given day [20]), with the net effect of decreasing the effectiveness of the established measures. Critical for the adherence to the decisions taken, to limit the diffusion of an infection, is that the scope and the characteristics of the surveillance, be explained fully and in detail [21].

It is also clear that respect for privacy is an unavoidable right of every individual and this should also be observed when considering public health, but sometimes it must be avoided to inform about the spread of the infection, as well as when contacting an individual infected is identified and notified to a third party. However, in exceptional circumstances, it is generally accepted to compromise individ- ual rights and freedoms without explicit subjects allowing the good of society to be achieved [22]. Furthermore, it has been repeatedly demonstrated that the speed of transmission of information relating to the spread of a communicable disease is essential, to implement the measures capable of contrasting the diffusion of the disease, especially in the first phase of the epidemic, when its speed is extremely high. We know that some delay passed before China informed the world of the serious coronavirus epidemic in its territory, with conceivable consequences.

Lockdown and quarantine seem at present the best solutions to limit the spread of SARS-CoV-2, however it is a very critical ethical point in public surveillance, because it limits the freedom of personal movements. Just for its hard characteristics, lockdown should be imposed only when clear, cogent needs are present, and its adoption would reasonably envisage a true benefit. This is what is now occurring in our country when several centers of infection have been identified. It is also thought that the quarantine should be adopted, when possible, in the less oppressive way and maintaining the provision for essential needs at least until more heavy measures could become necessary in order to improve the control of the infection. This is especially true when individuals already are in a condition of extreme misery, like homeless or immigrants who are more susceptible to any infection due to the poor hygienic and dietary habits and need particular attention and assistance. Obviously, lockdown and quarantine are differently experienced and endured according to the conditions of housing, being worst if in overcrowded lodgings, deprived of essential facilities. Even in Italy, where the access to NHS is relatively free, there could be barriers for homeless or immigrants due to lack of information, language barriers, literacy problems, fear to be identified and expelled.

Anyway, the more intriguing ethical problem regards the triage of patients with severe disease when it has to be decided who can be hospitalized and treated with intensive and semi-intensive respiratory care and who cannot. This situation arises when the request for hospitalization and treatment with oxygen and mechanical ventilation (invasive and non-invasive) overwhelms the resources of the health system. The NPIs described above are also intended to reduce this demand, if possible, by flattening the curve of the epidemic, thus reducing the stress of healthcare facilities. However useful as NPIs may be, it is always possible that at least in some regions or areas the supply of healthcare facilities is quickly exceeded. Of course, the problem of the availability of hospital beds and intensive care not only concerns COVID-19 patients, but also the many other patients who need hospitalization at the same time, due to non-COVID-19 emergencies. Deciding who can be admitted and who cannot often entail the difference between survival and death and this is always a hard decision, especially if age would be the only guide. Even worse if the age cut-off would vary according to the shortage of beds. However, others believe that short-term survival chances (e.g., actual disease), as well as long-term survival chances (e.g., co-morbidities, if any), should be considered [23]. In any case, it is not fair to add the stress of making those decisions to clinicians already overwhelmed by being in the first line, therefore triage algorithms (and "triage officers") have been proposed to allocate resources. Nevertheless, the first line will always be the place where these algorithms meet the real person and the past experience has not been so satisfactory (see over). It is obvious that the greater the lack of intensive care and intermediate care beds is the greater will be the stress on health staff.

\section{Organizational considerations - the role of pulmonary units}

To cope with the SARS-CoV-2 epidemic - and indeed with any 
other epidemic - a planned organization is needed first. This is not only based on what has been explained in the previous paragraphs, but it is also important to prepare plans for the best and most advantageous use, in terms of costs-benefit, of the respiratory medicine structures, both in hospital and extra-hospital settings. The 2009 document cited at the beginning [3] was produced to shape this use. Let us see now how much of its advice could be useful also for today.

The document stated that when the outbreak begins (especially if the first signs of the outbreak have been disregarded) and the NPIs have been implemented, non-urgent activities can be postponed and cancellations and postponements of routine activities can be devised, in order to devote all resources to the epidemic. A simple algorithm has been suggested to decide the urgent allocation of patients: patients who seek medical advice or who go to healthcare facilities with symptoms suggesting the disease A (H1N1) are evaluated (triage). If there are no signs of respiratory impairment, the patient is sent home after an evaluation of his/her risk profile. The use of Modified Early Warning Score (MEWS) [24], was recommended for this purpose. If the MEWS was greater than 4, the document suggests that the patient is sent to the A\&E department, possibly on a separate lane. Therefore, triage aimed to place the patient in one of four classes should be performed. The first one deserves the usual care (oxygen and supportive therapy and observation). The second one is for those who breathe spontaneously but need non-invasive ventilation. The third one is for those who need intensive care unit (ICU) for severe respiratory failure (measured mainly by blood gases analysis) or multi-organ failure. Finally, a subset of patients who need ECMO.

What was designed in this past document can also be applied today, with some changes: the respiratory specialists and respiratory units (these latter somehow specific to Italy where Pulmonary Units are planned on a basis of one unit per 400,000 to 800,000 inhabitants) can play a pivotal role. They can follow the first-class patient (referring to the above-mentioned triage classes; Table 1) at home (if possible with tele-medicine and in networking with GPs and other territorial level health personnel) [25]. Home care is predictable both for those who experience mild symptoms, which do not require hospitalization, and for those discharged from hospitals, because they have improved. This kind of patients must be monitored every $24 \mathrm{~h}$, but can also contact the nurse or specialists at any time, to communicate their situation. The pulmonologist can also collaborate with the medical departments to lead and plan assistance for hospitalized patients with non-severe respiratory problems (second class). The pulmonary unit can take care of patients hospitalized for respiratory failure, that does not require invasive mechanical ventilation (third class) and patients discharged from the ICU, regardless if they underwent ExtraCorporeal Membrane Oxygenation (ECMO) or not (patients coming from the two last classes), because they have improved and breath spontaneously. Pulmonary unit in this case plays a role of intermediate care unit [26]. Some anecdotal and confidential unpublished reports show that these intermediate care units operated successfully during the present emergency treating even older patients with non-invasive ventilation, if there were no enough ICU beds. Other reports refer of significant cases, beyond the uncontrolled rumors, of an epidemic hidden among the guests and the health personnel of the nursing homes.

The document mentioned previously [3] concerning the recommendations proposed for the A (H1-N1) outbreak, was never implemented because the 2009 pandemic, fortunately, did not spread throughout Italy. In other countries, like US and Canada, during the same period a triage protocol had been implemented and the results were not completely satisfying, independently from the triage tool used [27-29].

This time in Italy, as the outbreak started, in some regions there was no time to implement what the document recommended and, indeed, to use any type of planned actions, except those for emergency management. However, since in some Italian regions SARS$\mathrm{CoV}-2$ is fortunately far from being widespread, data are being collected as for the practical and ethical problems arising from the current situation. Anyway, at least in the Northern regions it was demonstrated that organizational failures directly cause or worsen ethical problems to health staff. So, mending organizational failures will reduce the ethical burden to health professionals

\section{Lessons to be learned}

So far, the COVID-19 epidemic has already given us some lessons that we can consider.

Outside the medical domain there is the problem created by the splitting of the NHS into 21 small regional health systems. This fragmentation has created not only inconsistencies in the management of the epidemic, but also confusion in public opinion, which in an emergency like the present one should absolutely be avoided. As a fall out of this "regionalization" in the past some regions have chosen to let private subjects provide health services to public payers. As a matter of fact, the region where this choice has been more pushed is the region where the pandemic bit far more (Lombardy). Only when we have data about the course of the pandemic in Italy we could understand if and to what extent this choice had a role in worsening the epidemic but observed facts are to be pondered about. This is a political problem and, although not qualified to address it in the present context, as health experts we believe that this issue will need to be debated on due time.

First of all, it is necessary to collect and process data about what happened and which were the most important drivers of the disease. To what extent social inequalities played a role, for instance, because so far we do not know if homeless, migrants and generally speaking poor people and hosts of retirement homes have had a worst outcome from the disease.

Among medical problems, the first lesson is the limit of hospital-centered care. There is no doubt that to reduce the danger of spreading the virus and increase the chances of successfully treating patients who require non-invasive and invasive ventilation, every effort should be made to keep mildly affected patients and all suspected cases out of hospitals. Consequently, a network of care

Table 1. Triage aims to place patients in one of the four classes.
1) Patients suitable for home care (mild symptoms, reliable caregivers)
2) Patients with usual care (oxygen and supportive therapy) in Medicine ward, with a short period of observation
3) Patients who breathe spontaneously but need Non-Invasive Ventilation (Pulmonary Unit)
4a) Patients who need Intensive Care Unit (ICU) for severe respiratory failure and/or multiple organ failure
4b) Patients who need ECMO 
facilities should be implemented at the territorial level, linking GPs, pulmonologists and specialized nurses. Once created, these networks obviously can also carry out respiratory consultations, exams and home care during non-epidemic periods. Someway, the new organization of respiratory medicine in Italy could rebuild (with obvious updating) the anti-tuberculosis network, which consisted of hospital beds and clinical networks.

Patients who require oxygen therapy (low $\mathrm{O}_{2}$ flow treatment) can also be cared for at home, provided that patients and caregivers are educated and reliable, that reliable tools for tele-monitoring can be provided and that patients, if necessary, can easily and quickly reach hospitals.

The second lesson is a double one: it means that in Italy i) there are too few pulmonologists, and ii) The Respiratory Medicine Units role is widely misunderstood. Italian NHS has already recognized the great importance of thoracic endoscopy. Now it is time to recognize also the importance of semi-intensive care and critical patient respiratory monitoring; in fact, each hospital should have in its team respiratory physicians, just as anesthesiologists are provided. There is also a need of implementing respiratory medicine activities at the territorial level as stated before: a network led by pulmonologist is necessary to take care of chronic patients as well as post-acute ones.

It is not (or not only) a problem of dedicated hospital beds but of staff. Easy as it is today to set a non-invasive ventilator, ventilating a patient is a difficult task and experience is needed for physicians as well as for nurses. It is true that not all the Italian pulmonologists are skilled in ventilating and monitoring critical patients (as not all the anesthesiologists are skilled in Intensive Care), but they have the education about respiratory function that makes learning of these issues easier and quicker.

Education and training are necessary for the young generations. So not only the number of less than 90 students entering the Respiratory medicine specialty schools at the national level (evidently underscored) should be re-modulated. In addition, the planned curriculum should be revised. University cannot cope alone with this task. Since a practical experience on a great number of cases is required according to the EU rules and ERS recommendations [30], hospitals with semi-intensive care sections should be part of the educating network as well as territorial level facilities for teaching home care essentials.

The third lesson concerns preparation for another (if any) similar emergency [2,31]: besides education and skills already mentioned, there is a problem of structures and devices. During the first weeks of February 2020, in some Italian regions, it was proposed to reopen old hospitals and actually whole small hospitals have been dedicated to COVID patients. A large number of ventilators and respiratory devices have been invested and many young graduates and doctors experienced in other specialties have been employed.

The question is now what to do in the aftermath when facilities should return to the former service. One first question is: how and where ventilators and all other devices used (and possibly to be used in the future) for the emergency can be stored and maintained with periodical maintenance? One possible answer is that all devices should be placed in containers. Containers can easily be stored and can be quickly mobilized, when necessary. But a more intriguing question is what to do for physicians?

Our idea is to use the usual flu period as a "school" for basic training and retraining. All doctors who work in respiratory, or general medicine units, know very well that there is a moderate to severe deficiency in hospital beds in the period from December to the end of February (or March). During this period, beds of the whole hospital and sometimes the aisles are occupied for respiratory patients [32], just like in other Countries, namely UK [33]. Consequently, this period can be used to train nurses and doctors of units other than Pulmonary or Internal Medicine units, to manage respiratory patients, with a certain interruption of normal activities such as elective surgical ones. Alternatively, staff can offer a short retraining period once a year. It is particularly important because planning interventions costs less than implementing them in an emergency, an event that nobody can rule out.

Finally, to emphasize that extra-hospital medicine (such as the GPs) should be continuously and effectively updated, trained on the behaviors and actions to be followed in "epidemic medicine", and supplied with the required protections.

\section{Conclusions and future directions}

SARS-CoV-2 will continue to circulate during this and the following years. When the NPIs are reduced, there will probably be a rebound of cases and, in any case, with the winter season, SARS$\mathrm{CoV}-2$ will circulate with the usual influence (A and B), as it was the case in 2009-2010 [4], until an effective vaccine will be widely produced and administered and herd immunity is sufficiently widespread. Once again NPIs will be needed and careful and constant vigilance by the aforementioned territorial level network will be required, aimed at grasping the start of a new epidemic from the very beginning, ignoring any political issue. Masks should be prescribed to everybody in community settings at least until vaccine will be ready and have shown its effectiveness. In fact, since it has been shown that asymptomatic infected persons could transmit the virus (and with a high viral load too) this is the only way to discontinue the transmission [34].

In a few months, we will have some information about the current pandemic in Italy and this will be useful for careful planning of the future, both updating the cited 2007 guidelines [2] and making the best use of the existing respiratory units. Actually, the first holistic immunological model of COVID-19 and the implications for prevention, diagnosis, and public health measures has been quite recently published [35]. In the short term, recruitment is necessary of retired pulmonologists for backlog, both in the clinic and in surgery. Allocation of the remaining devices to internal medicine and respiratory departments, according to the evolution of the first phase of the pandemic. In the medium term, training and re-training of respiratory specialists (when necessary) and periodic training of other personnel as mentioned above.

In the long term, more careful planning of the number of Pulmonary specialists and Respiratory medicine facilities is needed to comply with ordinary activities and to establish a network for the management of usual chronic respiratory conditions. A network between hospitals and territorial health services (respiratory and non-respiratory specialists, and GPs) will be useful for facing another future pandemic.

\section{Acknowledgements}

Special thanks to Carlo Favaretti, MD, MPH (Centre for Leadership in Medicine, Catholic University of the Sacred Heart, Rome) for having reviewed this paper and making useful suggestions.

\section{References}

1. Pisano GP, Sadun R, Zanini M [Internet]. Lessons from Italy's response to Coronavirus. Harvard Business Review 2020 Mar 27. Available from: https://hbr.org/2020/03/lessons-from-italysresponse-to-coronavirus

2. Centro nazionale per la Prevenzione e il Controllo delle Malattie 
del Ministero della Salute (CCM). Piano nazionale di preparazione e risposta a una pandemia influenzale. 2007. Available from: https://www.epicentro.iss.it/focus/flu_aviaria/pdf/pianopandemico.pdf

3. Nardini S, De Benedetto F, Donner CF, Sanguinetti CM, Conte $\mathrm{S}$, Marino L, et al. [AIMAR protocol for the management of pandemia influenza $\mathrm{A}(\mathrm{H} 1 \mathrm{~N} 1)-2009$ in respiratory divisions. Role and competence of the Pneumo(phtysio)logy department]. [Article in Italian]. Multidiscip Respir Med 2009;4: 434-43.

4. LaRussa P. 2009 pandemic novel H1N1 flu: what have we learned? Semin Respir Crit Care Med 2011;32:393-9.

5. WHO. Rolling updates on coronavirus disease (COVID-19). Accessed on March 2020. Available from: https://www. who.in/emergence/diseases novelcoronavirus-2019 .

6. Task force COVID-19 del Dipartimento Malattie Infettive e Servizio di Informatica Istituto Superiore di Sanità. Infografica. Accessed on: 5 April 2020.

7. WHO. Coronavirus disease 2019 (COVID-19). Situation Report - 77. Accessed on: 6 April 2020.

8. Atluri S, Manchikanti L, Hirsch JA. Expanded umbilical cord mesenchymal stem cells (UC-MSC) as a therapeutic strategy in managing critically III COVID-19 patients: The case for compassionate use. Pain Physician 2020;23:E71-83.

9. Li R, Pei S, Chen B, Song Y, Zhang T, Yang W, et al. Substantial undocumented infection facilitates the rapid dissemination of novel coronavirus (SARS-CoV-2). Science 2020; pii: eabb3221. doi: 10.1126/science.abb3221. [Epub ahead of print].

10. Day M. Covid-19: four fifths of cases are asymptomatic, China figures indicate. BMJ 2020;369:m1375. doi: 10.1136/bmj. m1375. [Response available from: https://www.bmj.com/content/369/bmj.m1375/rr-5].

11. Zhu L, Li C, Ning SS, Chen S, Cao L, Yang GJ, et al. [Epidemiological characteristics of COVID-19 in Shaanxi province].[Article in Chinese with English Abstract]. Zhonghua Liu Xing Bing Xue Za Zhi 2020 41:E034. doi: 10.3760/cma.j.cn112338-20200225-00172. [Epub ahead of print].

12. Singhal T. A review of Coronavirus Disease-2019 (COVID-19). Indian J Pediatr 2020;87:281-6.

13. Senior Italia FederAnziani. Proceedings Eighth National Congress. 21-22 Novembre 2019. Rimini, Italy.

14. Ferguson N, Laydon D, Nedjati-Gilani G, Imai N, Ainslie K, Baguelin M, et al. Imperial College COVID-19 Response Team. Report 9: Impact of non-pharmaceutical interventions (NPIs) to reduce COVID19 mortality and healthcare demand, 2020 Mar 16. Available from: https://spiral.imperial.ac. uk:8443/handle/10044/1/77482

15. [Internet]. Corona Virus - Covid19 - Covid Trends. Accessed on: 17 April 2020. Available from: https://covidtrends.com/

16. Langmuir AD. The surveillance of communicable diseases of national importance. N Engl J Med 1963;268:182-92.

17. Langmuir AD. Evolution of the concept of surveillance in the United States. Proc R Soc Med 1971;6:681-4.

18. Selgelid MJ. Ethics of infectious disease control. In: S.R. Quah, editor. International Encyclopedia of Public Health 2nd ed.
Academic Press; 2017; p. 11-16.

19. Gostin LO. Public health surveillance and research. Personal privacy and the "right to know". In: Public health law. Power, duty, restraint. California University Pres; 2008; p. 287-330.

20. Ministero dell'Interno. Coronavirus, i dati dei servizi di controllo. Accessed on: 6 April 2020. Available from: https://www.interno. gov.it/it/coronavirus-i-dati-dei-servizi-controllo

21. Petrini C. Ethics in public health surveillance. Ann Ist Sup Sanità 2013;49:347-53.

22. Lee LM, Heilig CM, White A. Ethical justification for conducting health surveillance without patient consent. Am J Public Health 2012;102:38-44.

23. Rosenbaum L. Facing Covid-19 in Italy - Ethics, logistics and therapeutics on the epidemic's front line. New Engl J Med 2020. doi: 10.1056/NEJMp2005492.

24. Gardner-Thorpe J, Love N, Wrightson J, Walsh S, Keeling N. The value of Modified Early Warning Score (MEWS) in surgical in-patients: a prospective observational study. Ann R Coll Surg Engl 2006;88:571-5.

25. WHO. Assistance at home for patients with suspected new coronavirus infection (COVID-19) who exhibits mild symptoms and management of their contact. Intermediate guide. 4 February 2020.

26. Waydhas C, Herting E, Kluge S, Markewitz A, Marx G, Muhl E, et al. Intermediate care units: Recommendations on facilities and structure. Med Klin Intensivmed Notfmed 2018;113:33-44.

27. Christian MD, Hamielec C, Lazar NM, Wax RS, Griffith L, Herridge MS, et al. A retrospective cohort pilot study to evaluate a triage tool for use in a pandemic. Crit Care 2009,13:R170.

28. Khan Z, Hulme J, Sherwood N. An assessment of the validity of SOFA score based triage in H1N1 critically ill patients during an influenza pandemic. Anaesthesia 2009;64:1283-8.

29. Fink SL. Worst case: rethinking tertiary triage protocols in pandemics and other health emergencies. Crit Care 2010;14:103.

30. Loddenkemper R, Séverin T, Mitchell S, Belevskiy A, Chuchalin A, de Hosson S, et al. Adult HERMES: criteria for accreditation of ERS European training centres in adult respiratory medicine. Breathe 2010;7:170-88. doi: 10.1183/20734735.020910.

31. Li JY, You Z, Wang Q, Zhou ZJ, Qui Y, Luo R, et al. The epidemic of 2019-novel- coronavirus (2019- nCoV) pneumonia and insights for emerging infectious diseases in the future. Microbes Infect 2020;22:80-5.

32. Del Donno M, Di Sorbo A, Micco A. Overcrowding in hospital emergency departments in winter and increase in beds in Respiratory disease units. Rass Patol App Resp 2018;33:206-14.

33. British Lung Foundation. Out in the cold: lung disease, the hidden driver of NHS winter pressure. December 2017. Available from: https://www.blf.org.uk/policy/out-in-the-cold

34. Leung CC, Lam TH, Cheng KK. Mass masking in the COVID19 epidemic: people need guidance. Lancet 2020;395:945.

35. Matricardi PM, Dal Negro RW, Nisini R. The first, holistic immunological model of COVID-19. Implications for prevention, diagnosis, and public health measures. Pediatr Allergy Immunol 2020. doi: 10.1111/pai.13271. [Epub ahead of print].

Received for publication: 29 April 2020. Accepted for publication: 19 May 2020.

This work is licensed under a Creative Commons Attribution-NonCommercial 4.0 International License (CC BY-NC 4.0).

(C) Copyright: the Author(s), 2020

Licensee PAGEPress, Italy

Multidisciplinary Respiratory Medicine 2020; 15:672

doi:10.4081/mrm.2020.672 